\title{
The theory of functional equations in high school education
}

\author{
DOROTTYA BESSENYEI
}

\begin{abstract}
In this paper, we are going to discuss some possible applications of the theory of functional equations in high school education. We would like to line up some problems, the solution of which by functional equations are mostly not new results - they have also been treated in [1] and [2] -, although their demonstrations in high school can show a new way in teaching of talented students. The area of the rectangle, the calculating method of compound interest, binomial coefficients, Euler's formula, the scalar product and the vector product of vectors - we are looking for the reasons behind the well-known formulas. Finally, we are going to give a functional equation in connection with mean values. It can be understood easily, but its solution is beyond the high school curriculum, so we advise this part only to the most talented students.
\end{abstract}

Key words and phrases: functional equations, high school education, area of the rectangle, compound interest, binomial coefficient, Euler's formula, scalar product, vector product, arithmeticgeometric mean.

ZDM Subject Classification: D20, G70, I70.

\section{Introduction}

In the beginning of the 21 st century, it is worth thinking over whether the conventional teaching methods could be applied successfully in the future. The school is already not the obligate stage of getting information. Can it play indispensable role in transmission of knowledge henceforward? In our opinion it can, if it considers as its task not only to communicate the facts, but to concentrate on to show the deepness of global contexts and problems with the help of teachers, who have excellent professional knowledge.

Copyright (c) 2012 by University of Debrecen 


$$
\text { "tmcs-bessenyei" — 2012/11/28 — 11:56 — page 346 — \#2 }
$$

Unfortunately, nowadays the mathematical education on intermediate level contains less and less proofs, justification, explanation in Hungary. Together with the increasing curriculum, the teaching process moved to a more mechanical way. According to Ambrus [3], the psychological base of this tendency is the so-called behaviorism, the famous representative of which was Skinner (1904-1990). The followers of this school did not imagine the learning process to be constructive but to be reproductive: break the material down into its small component parts and drill the students till we achieve the desired result. They considered mathematics only as a system of ideas and rules, and people as machines which can be programmed. The situation has not been so extreme in Hungary, however the tendency shows this forwards, because less and less proofs of theorems are obligatory, less and less exercises are set centrally, which urge students on deeper thinking. Naturally there is no way to mention the motivation of the ideas. The students have to accept the definitions without knowing the deeper mathematical background. Fortunately there are students who ask questions and are curious to know the answers, hereby trying the teacher's knowledge.

It often occurs that we get our information of the nature through experimental way. We get to know the gravitation before we could realize the specific physical background. We know that the leaves of plants are green before we could understand the process of photosynthesis. The initial purpose of teaching may be to condition the facts, however students in advanced level have many questions and require the exact explanations. This paper would like to make an attempt at giving these answers with the help of functional equations among those pupils who are studying for final examination in advanced level. Hence they know the significant part of high school curriculum and the fundamental categories of analysis like limit of sequences and functions, continuity and differentiability of functions, integral, etc.

The theory of functional equations is just the point which could clarify the origin of certain connections. The purpose is not to make students understand difficult demonstrations, but to give them instruments for discovering the deeper layers of mathematics.

\section{Possible applications in high school}

In first approximation, the functional equations and the problems in connection with them are very far from the thinking of high school students in Hungary. Among other things it is because we often have to apply functions in several variables, and the students meet functions only in single variable. Working in the three dimensional space we fix one variable to get for example the formulas for the body of revolution. On the other hand 
it is not usual to speak about a function without the knowledge of its formula moreover its type. There are only a few tasks where the students have to infer the nature of the function by its graph. The experience shows that this is more difficult than conversely, namely, to characterize a given function and draw its graph.

On the other hand, according to our experience, the talented students can easily get used to this new treatment. Moreover, they enjoy to create the solutions in interactive way.

Throughout this paper, $\mathbb{N}, \mathbb{Z}$, and $\mathbb{R}^{+}$shall denote the sets of positive integers, integers and positive real numbers, respectively.

\subsection{The computing of the area of the rectangle}

Although this problem is found in [2], in this paper we give a new treatment of it avoiding the concept of continuity. We can work out this apparently simple task with the help of CAUCHY's (1789-1857) equation

$$
f(x+y)=f(x)+f(y)
$$

the solutions of which will be called additive functions. Denote the value of the area by $T(x, y)$, because we suppose that the area depends only on the lengths $x$ and $y$ of the sides. Moreover, we would like to divide side $x$ into the parts $x_{1}$ and $x_{2}$, so that the sum of the two given rectangle's area be equal to the area of the initial rectangle, as Figure 1 shows. The same thing has to be true for side $y$. That means, our function has to be additive in both variables. Furthermore, the area of the unit square is supposed to be 1 .
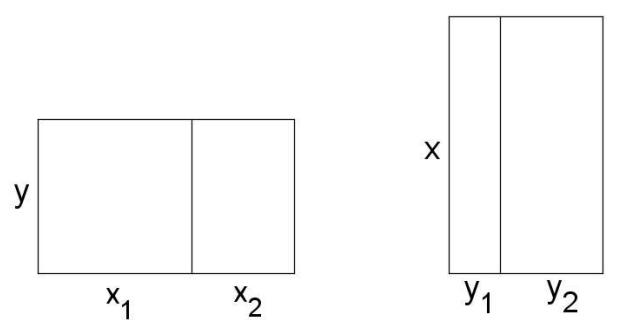

Figure 1

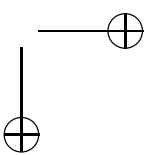


In short, we are looking for a function $T: \mathbb{R}^{+} \times \mathbb{R}^{+} \rightarrow \mathbb{R}^{+}$satisfying the properties

$$
\begin{array}{rlrl}
T\left(x_{1}, y\right)+T\left(x_{2}, y\right) & =T\left(x_{1}+x_{2}, y\right) & & \left(x_{1}, x_{2}, y \in \mathbb{R}^{+}\right) \\
T\left(x, y_{1}\right)+T\left(x, y_{2}\right) & =T\left(x, y_{1}+y_{2}\right) & & \left(x, y_{1}, y_{2} \in \mathbb{R}^{+}\right) \\
T(1,1) & =1 . &
\end{array}
$$

If we consider $y$ to be fixed, by (1), we get the Cauchy equation, that is, we have an additive function $f: \mathbb{R}^{+} \rightarrow \mathbb{R}^{+} ; x \mapsto T(x, y)$. This is why for arbitrary $n \in \mathbb{N}$

$$
f(n x)=n f(x),
$$

furthermore

from where

$$
f\left(\frac{1}{n} x\right)=\frac{1}{n} n f\left(\frac{1}{n} x\right)=\frac{1}{n} f\left(n \frac{1}{n} x\right)=\frac{1}{n} f(x),
$$

$$
f\left(\frac{m}{n} x\right)=m f\left(\frac{1}{n} x\right)=\frac{m}{n} f(x)
$$

in case of $m \in \mathbb{N}$. Accordingly, for positive rational $r$, we have

$$
f(r x)=r f(x) .
$$

Our function $f$ is positive by definition, and increasing because of

$$
f(x+y)=f(x)+f(y)>f(x) \quad\left(x, y \in \mathbb{R}^{+}\right) .
$$

Approximate $x$ from below and above with sequences $\left\langle r_{n}\right\rangle$ and $\left\langle R_{n}\right\rangle$ of rational terms. For $n \in \mathbb{N}$ and $x \in \mathbb{R}^{+}$, we have

$$
[n x] \leq n x<[n x]+1
$$

where $[n x]$ denotes the integer part of $n x$, i.e., $[n x]$ is the greatest integer not greater than $n x$. Therefore

i.e.,

$$
\frac{[n x]}{n} \leq x<\frac{[n x]}{n}+\frac{1}{n},
$$

$$
0 \leq x-\frac{[n x]}{n}<\frac{1}{n}
$$

so $\frac{[n x]}{n}$ tends to $x$ as $n$ tends to infinity.

Thus, for the $n$-th terms

$$
r_{n}=\frac{[n x]}{n} \text { and } R_{n}=\frac{[n x]}{n}+\frac{1}{n},
$$


of the sequences $\left\langle r_{n}\right\rangle$ and $\left\langle R_{n}\right\rangle$, with the notation $c=f(1)$, we get that

$$
c \cdot r_{n}=r_{n} \cdot f(1)=f\left(r_{n}\right) \leq f(x) \leq f\left(R_{n}\right)=R_{n} \cdot f(1)=c \cdot R_{n} .
$$

Since $\left\langle r_{n}\right\rangle$ and $\left\langle R_{n}\right\rangle$ tend to $x$, we get

$$
f(x)=c \cdot x,
$$

if $x \in \mathbb{R}^{+}$. The constant $c$ may depend on the fixed $y$.

So we obtain from Cauchy's equation that $T(x, y)=c(y) x$, whence, by (2), we get that $c$ is additive, too. Since $T(x, y)>0$, with the above order of ideas, there exists a constant $\left(c_{0}>0\right)$ again that $c(y)=c_{0} y$. Thus $T(x, y)=c_{0} x y$, and by $(3), c_{0}=1$. Finally, we get the desired formula

$$
T(x, y)=x y \text {. }
$$

It is to be noted that raising the question is linked with the name of LEGENDRE (1791), the precise treatment was worked out by DARBOUX (1880).

\subsection{The calculating method of compound interest}

In high school education, the compound interest is the most frequently occurrent example for applying the geometric sequence, as we can see in [7]. Denote the initial capital by $T_{0}$, the number of the unit of time passed by $n(\in \mathbb{N})$ and the percent of interest in unit time by $p$, respectively. The calculation of the $n$-th term $T_{n}$ is a standard exercise. Surprisingly, behind the known formula

$$
T_{n}=T_{0} \cdot\left(1+\frac{p}{100}\right)^{n}
$$

the Cauchy equation is again.

Let us see the more general composition of the problem. We are looking for the two-variable functions which give, how much capital we will have from the capital of quantity $x$ within time $t$ (this will be denoted by $F(x, t)$ ). The following conditions seem to be natural:

$$
\begin{aligned}
& F(x+y, t)=F(x, t)+F(y, t) \quad\left(x, y, t \in \mathbb{R}^{+}\right) \\
& F(x, t+s)=F(F(x, t), s) \quad\left(x, t, s \in \mathbb{R}^{+}\right),
\end{aligned}
$$

where $F$ is monotonic in the second variable and has only positive values. Equation (4) expresses that $F$ is additive in the first variable, while equation (5) shows that if we invest 
a capital of quantity $x$ for time $t$, then this new capital for further time $s$, this is the same as if we invested the origin capital of quantity $x$ for time $t+s$.

If we consider the first variable, equation (4) is analogous to the Cauchy equation. Applying the method, we used by computing the area of the rectangle, we have that there exists a function $c: \mathbb{R}^{+} \rightarrow \mathbb{R}^{+}$such that $F$ has the form

$$
F(x, t)=c(t) x
$$

Replacing it into (5), we get $c(t+s) x=c(s) F(x, t)=c(s) c(t) x$ which implies that

$$
c(t+s)=c(t) c(s)
$$

namely $l n c$ is a monotonic additive function. Therefore there exists $c_{0} \in \mathbb{R}$ such that $\ln c(t)=c_{0} t$, whence $c(t)=e^{c_{0} t}$ follows. Finally, with the definition $e^{c_{0}}:=q$, we obtain that

$$
F(x, t)=x q^{t}
$$

for all values $x>0$ and $t>0$.

\subsection{Binomial coefficients}

Solving the exercises of combinatorics can be often a great challenge for students. There is a theme again, within it is not possible to apply the learned formulas mechanically. Approach to the problem from many sides can help the understanding: describe for example the combination without repetition by a functional equation.

Therefore we consider a set with $n$ objects, from which we would like to select $k$ objects without caring about the order in which they are chosen $(n, k \in \mathbb{N}, k \leq n)$. Denote the number, which gives in how many different ways can we take the above selection, by $f_{n}(k)$. After fixing the value $n \in \mathbb{N}$, we may write simply $f(k)$, where $f:\{0,1, \ldots, n\} \rightarrow \mathbb{R}$.

Besides the trivial condition $f(0)=1$, we would like to set up a connection between $f(k)$ and $f(k+1)$. It is not difficult to think over that all to the sets, stay from $k$ pieces of objects, we can choose $(n-k)$ pieces to get sets from $(k+1)$ pieces of objects. Still we have to take care, because we enumerated the sets of $(k+1)$ elements $(k+1)$ times. Thus we have the functional equation

$$
f(k+1)=\frac{n-k}{k+1} f(k) \quad f:\{0,1, \ldots, n-1\} \rightarrow \mathbb{R},
$$

from where

$$
(n-k) f(k)=(k+1) f(k+1) .
$$


If we write $0,1,2, \ldots, k-1$ in place of $k$, we get

$$
\begin{aligned}
n f(0) & =1 \cdot f(1) \\
(n-1) f(1) & =2 \cdot f(2) \\
(n-2) f(2) & =3 \cdot f(3) \\
& \vdots \\
(n-(k-1)) f(k-1) & =k \cdot f(k) .
\end{aligned}
$$

Multiplying both sides of the above equations, we have

$$
\begin{gathered}
n(n-1)(n-2) \cdots(n-(k-1)) \cdot f(0) f(1) f(2) \cdots f(k-1)= \\
=1 \cdot 2 \cdot 3 \cdots k \cdot f(1) f(2) \cdots f(k-1) f(k) .
\end{gathered}
$$

Completing the possible simplifications, it follows that

$$
\frac{n !}{(n-k) !}=k ! \cdot f(k)
$$

that is,

$$
f(k)=\frac{n !}{k !(n-k) !}=\left(\begin{array}{l}
n \\
k
\end{array}\right),
$$

which is just the desired binomial coefficient $(n, k \in \mathbb{N}, k \leq n)$.

We raise the question: are there functional equations for other concepts of combinatorics, too? We leave the answering to the Reader.

\subsection{Euler's formula for planar graphs}

In Hungary, the high school curriculum in intermediate level contains the fundamental categories of graph theory. Students have to know the definition of planar graph, too. This is a graph that can be embedded in the plane, i.e., it can be drawn in such a way that no edges cross each other.

We are looking for a connection among the number of the graph's vertexes, edges and regions (bounded by edges). Denote the function, which gives the number of the graph's regions $(r)$, by $f_{v}(e)$, where $v$ and $e$ are the number of vertexes and edges, respectively. If we fix the number of vertexes $(v \geq 2)$, we get the function $f:\{1, \ldots, n\} \rightarrow \mathbb{N}$. In this case, we can easily illustrate the equation $f(e+1)=f(e)+1$, that is, adding one more edge to the graph results one more region. Iterating this process, we have that $f(e+c)=f(e)+c$, where $c$ is a constant $(c \in \mathbb{N}$ and $c \geq v-1)$. According to this fact,

$$
f(e)=f(v-1+(e+1-v))=f(v-1)+(e+1-v) .
$$


We know furthermore that $f(v-1)=0$, because in the case of $e=v-1$, we get the maximal connected graph without circles, so this graph has not any region. Replacing it into the above equation, we get that

$$
r=f(e)=e+1-v,
$$

that is, $v-e+r=1$. If we add to the regions the outer, infinitely-large region, and we call those together faces, we get the real Euler's formula:

$$
v-e+f=2,
$$

which is true also for polyhedrons ( $v, e$ and $f$ are the number of vertexes, edges and faces of it).

\subsection{The scalar product and the vector product of vectors}

On hearing the definition of scalar and vector multiplication, a lot of students ask the question rightly: why do we compute just like this? In order to be realized the desired properties of operations or to be able to compute with the coordinates easily? These are not adequate answers after all because they do not contain the requirement: why it cannot be otherwise.

Without knowing the formula, we start from the following natural conditions which the products have to satisfy. For precision's sake, we remark that for a rotation of the space, the scalar product is invariant and the vector product undergoes the same rotation. Furthermore, we have to know how to add two vectors and how to calculate the length of the resultant of those.

(a) Both multiplications are associative:

$$
\begin{gathered}
(\mathbf{a b}) \mathbf{c}=\mathbf{a}(\mathbf{b c}) \quad \forall \mathbf{a}, \mathbf{b}, \mathbf{c} \\
(\mathbf{a} \times \mathbf{b}) \times \mathbf{c}=\mathbf{a} \times(\mathbf{b} \times \mathbf{c}) \quad \forall \mathbf{a}, \mathbf{b}, \mathbf{c} .
\end{gathered}
$$

(b) Scalar factors can be taken out of any factor of the products:

$$
\begin{gathered}
(\lambda \mathbf{a}) \mathbf{b}=\lambda(\mathbf{a b})=\mathbf{a}(\lambda \mathbf{b}) \quad \forall \mathbf{a}, \mathbf{b} ; \lambda \in \mathbb{R} \\
(\lambda \mathbf{a}) \times \mathbf{b}=\lambda(\mathbf{a} \times \mathbf{b})=\mathbf{a} \times(\lambda \mathbf{b}) \quad \forall \mathbf{a}, \mathbf{b} ; \lambda \in \mathbb{R} .
\end{gathered}
$$

(c) Both multiplications are distributive:

$$
\begin{gathered}
(\mathbf{a}+\mathbf{b}) \mathbf{c}=\mathbf{a c}+\mathbf{b} \mathbf{c} \quad \forall \mathbf{a}, \mathbf{b}, \mathbf{c} \\
(\mathbf{a}+\mathbf{b}) \times \mathbf{c}=\mathbf{a} \times \mathbf{c}+\mathbf{b} \times \mathbf{c} \quad \forall \mathbf{a}, \mathbf{b}, \mathbf{c},
\end{gathered}
$$


(d) furthermore in the case of $|\mathbf{e}|=|\mathbf{f}|=1$ :

$$
\begin{aligned}
\mathbf{e e} & =1 \\
|\mathbf{e} \times \mathbf{f}| & =1 \quad \text { if } \mathbf{e} \perp \mathbf{f} .
\end{aligned}
$$

REMARK 1. We can show, by a calculation in [1, p.28], that these assumptions imply that

$$
\begin{array}{rlll}
\mathbf{a b}=0 & \text { if } & \mathbf{a} \perp \mathbf{b} \\
|\mathbf{a} \times \mathbf{b}|=0 & \text { if } & \mathbf{a} \| \mathbf{b} .
\end{array}
$$

By condition (b), we can consider the $\mathbf{a}, \mathbf{b}, \mathbf{c}$ unit coplanar vectors where $\mathbf{a}$ and $\mathbf{b}$ form the angle $2 \varphi, \mathbf{b}$ and $\mathbf{c}$ form the angle $\psi$ - see in Figure 2.

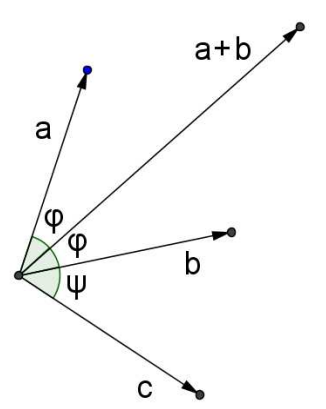

Figure 2

Denote the required product of two unit vectors having the angle $\alpha$ by $f(\alpha)$. Because of condition (c), we have that

$$
f(2 \varphi+\psi)+f(\psi)=2 \cos \varphi \cdot f(\varphi+\psi)
$$

where we used the formula for computing the length of the resultant. If we write the variables $x$ and $y$ in place of $\varphi$ and $\psi$ respectively, we obtain that

$$
f(2 x+y)+f(y)=2 f(x+y) \cos x .
$$

The role of the variables is interchangeable, so

$$
f(2 y+x)+f(x)=2 f(y+x) \cos y .
$$


If we write $(x-y)$ in place of the variable $x$, we get

$$
f(x+y)+f(x-y)=2 f(x) \cos y
$$

The solutions of this equation was found by JÁNOS ACZÉL applying the following method [1, p.24]. By replacing the variable pair $(x, y)$ in three ways

$$
\begin{aligned}
(x, y) & =(0, t) \\
(x, y) & =\left(\frac{\pi}{2}+t, \frac{\pi}{2}\right) \\
(x, y) & =\left(\frac{\pi}{2}, \frac{\pi}{2}+t\right),
\end{aligned}
$$

respectively, together with the notations $f(0):=p$ and $f\left(\frac{\pi}{2}\right):=q$ he got the following three equations $(p, q \in \mathbb{R})$ :

$$
\begin{aligned}
f(t)+f(-t) & =2 p \cos t \\
f(t+\pi)+f(t) & =0 \\
f(t+\pi)+f(-t) & =2 q \cos \left(\frac{\pi}{2}+t\right)=-2 q \sin t .
\end{aligned}
$$

Subtracting the third one from the sum of the first two equations, we get that

$$
2 f(x)=2 p \cos x+2 q \sin x .
$$

After simplification, we find that the general form of the equation is

$$
f(x)=p \cos x+q \sin x \quad(x \in] 0, \pi[) .
$$

In the case of the scalar product, by condition $(\mathrm{d}), f(0)=1$, so $p=1$. Alike, $f(\pi / 2)=0$ because of Remark 1 , so $q=0$ follows, too. Accordingly the desired function is

$$
f(x)=\cos x \text {. }
$$

In the case of the vector product, through the condition (d), $f(\pi / 2)=1$, so $q=1$. Alike, $f(0)=0$ because of Remark 1 , so $p=0$ follows, too. Finally we found the function

$$
f(x)=\sin x
$$

Universally, applying the above calculations for arbitrary $\mathbf{a}$ and $\mathbf{b}$ vectors, there exist real numbers $\lambda$ and $\mu$ such that

$$
\mathbf{a b}=\left(\lambda \mathbf{e}_{a}\right)\left(\mu \mathbf{e}_{b}\right)=\lambda \mu\left(\mathbf{e}_{a} \mathbf{e}_{b}\right)=\lambda \mu \cos \varangle(\mathbf{a}, \mathbf{b})
$$


where $\mathbf{e}_{a}$ is a unit vector having the same direction as $\mathbf{a}$ and $\mathbf{e}_{b}$ is a unit vector having the same direction as $\mathbf{b}$. We also know that $\lambda$ equals to $|\mathbf{a}|$ and $\mu$ equals to $|\mathbf{b}|$. We got the familiar form

$$
\mathbf{a b}=|\mathbf{a}||\mathbf{b}| \cos \varangle(\mathbf{a}, \mathbf{b}) .
$$

Similarly, for the vector product

$$
\mathbf{a} \times \mathbf{b}=\left(\lambda \mathbf{e}_{a}\right) \times\left(\mu \mathbf{e}_{b}\right)=\lambda \mu\left(\mathbf{e}_{a} \times \mathbf{e}_{b}\right)=\lambda \mu \sin \varangle(\mathbf{a}, \mathbf{b}) \mathbf{e}^{\prime}
$$

namely

$$
\mathbf{a} \times \mathbf{b}=|\mathbf{a}||\mathbf{b}| \sin \varangle(\mathbf{a}, \mathbf{b}) \mathbf{e}^{\prime}
$$

where $e^{\prime}$ is such a unit vector that $\mathbf{e}^{\prime} \perp \mathbf{a}, \mathbf{e}^{\prime} \perp \mathbf{b}$ and the vectors $\mathbf{a}, \mathbf{b}, \mathbf{e}^{\prime}$ form a righthanded system.

\section{A further equation in connection with mean values}

There are important themes which occur only superficially in high school education. For example, while teaching mean values lots of questions arise. At first, we have to decide with which subject we would like to connect them: relate them with geometrical problems, mention them at the time of teaching the arithmetic and geometric sequences, or use maybe as an instrument of statistical characterizations? Wouldn't it be better to open a separated chapter and introduce the students different kinds of means? In our opinion, it should be devoted greater care to show the fields of applications because it can enrich the instruments of solving exercises.

In high school, we differentiate four kinds of means of two positive numbers $a$ and $b$ : arithmetic, geometric, harmonic and square means (the last two ones are obligatory only in advanced level):

$$
\begin{gathered}
A(a, b)=\frac{a+b}{2} \\
G(a, b)=\sqrt{a b} \\
H(a, b)=\frac{2 a b}{a+b}=\left(\frac{a^{-1}+b^{-1}}{2}\right)^{-1} \\
S(a, b)=\sqrt{\frac{a^{2}+b^{2}}{2}}=\left(\frac{a^{2}+b^{2}}{2}\right)^{\frac{1}{2}} .
\end{gathered}
$$

At the same time, it is hidden for the students that all of them can be interpreted as special cases of a general concept, because, in the case of two positive integers, the "mean" name 
suggests only the fact that the third number - mapped to the first two - is between them if the two numbers are different and equals them if the two numbers are the same [8, p.5].

We get the following chain of inequalities among them:

$$
H \leq G \leq A \leq S
$$

which can be proved for example with the help of this picture of a trapezium [8, p.7], where $a$ and $b$ are the given positive numbers and $E_{i} F_{i}$ has the length of the corresponding mean, as it can be seen in Figure 3.

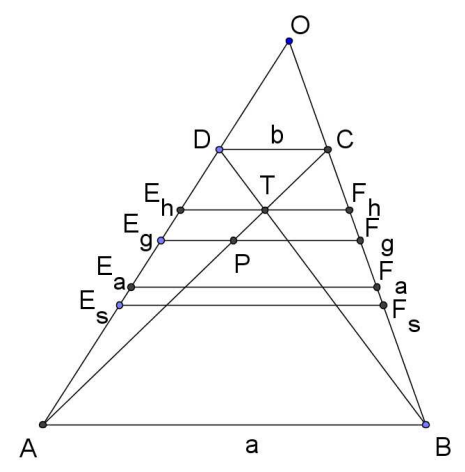

Figure 3

We have to notice the analogy in the formulas. We can give the harmonic, the arithmetic and the square mean in the same form:

$$
M_{p}(a, b)=\left(\frac{a^{p}+b^{p}}{2}\right)^{\frac{1}{p}}
$$

from where we get the arithmetic mean if $p=1$, the square mean if $p=2$ and the harmonic mean if $p=-1$. Call $M_{p}(a, b)$ the $p$-th power mean of the positive numbers $a$ and $b$. At first sight, the geometric mean cannot be fitted into the series, so we define it as the 0 -th power mean:

$$
M_{0}(a, b)=\sqrt{a b}
$$

In this way we can extend the explanation for an arbitrary integer $p$. Furthermore it can be proved that the $(p+1)$-th power mean of $a$ and $b\left(a<b ; a, b \in \mathbb{R}^{+}\right)$is not less than the $p$-th power mean of them. If we would like to illustrate the $p$-th power means 
in a coordinate-system, we have a series of points "twisted" between the lines $y=a$ and $y=b$. Bind these points in the hope that we can define the power mean for real exponent. One can see it in Figure 4.

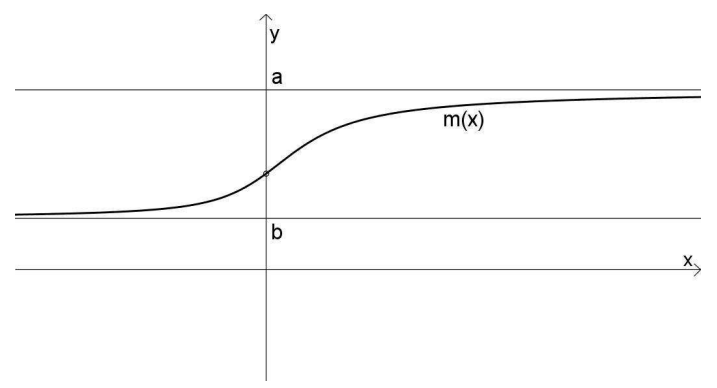

Figure 4

It can be proved with a longer calculation that function $M_{p}$ fills the gap in the above given case of $p=0$. Thus $M_{p}$ will be continuous if we choose $M_{0}(a, b)$ as $\sqrt{a b}$.

The explanation can be extended to $n(n \in \mathbb{N})$ positive numbers in the way that the mean shall be between the minimum and the maximum of the considered numbers. We can give a general definition for mean values:

DeFinition 1. Let $p \in \mathbb{R}, n \in \mathbb{N}$ and

$$
M_{p}\left(x_{1}, x_{2}, \ldots, x_{n}\right)= \begin{cases}\left(\frac{x_{1}{ }^{p}+x_{2}{ }^{p}+\cdots+x_{n}{ }^{p}}{n}\right)^{\frac{1}{p}} & \text { if } p \neq 0 \\ \sqrt[n]{x_{1} x_{2} \cdots x_{n}} & \text { if } p=0\end{cases}
$$

for $x_{1}, x_{2}, \ldots, x_{n} \in \mathbb{R}^{+}$. Then the value $M_{p}\left(x_{1}, x_{2}, \ldots, x_{n}\right)$ is called the $p$-th power mean of the $x_{1}, x_{2}, \ldots, x_{n} \in \mathbb{R}^{+}$numbers.

\subsection{The arithmetic-geometric mean}

The concept of sequence of real numbers is known for 12-grade students and the examination of their monotonicity and convergency is a requirement in advanced level. We mention the recursive property in connection with the famous Fibonacci sequence. Thus we know everything to define the following sequences $\left\langle a_{n}\right\rangle$ and $\left\langle b_{n}\right\rangle$ :

$$
\begin{array}{cc}
a_{0}:=a>0 & b_{0}:=b>0 \\
a_{n+1}:=\frac{a_{n}+b_{n}}{2} & b_{n+1}:=\sqrt{a_{n} b_{n}} .
\end{array}
$$




$$
\text { “tmcs-bessenyei” — 2012/11/28 - 11:56 — page 358 — \#14 }
$$

Obviously, the $(n+1)$-th term of the sequences is defined by the arithmetic and geometric means of the $n$-th terms: $a_{n+1}=A\left(a_{n}, b_{n}\right)$ and $b_{n+1}=G\left(a_{n}, b_{n}\right)$, respectively (see e.g. [5]). The following theorem and also its proof are found in [4, p.2].

THEOREM 1. The sequences $\left\langle a_{n}\right\rangle$ and $\left\langle b_{n}\right\rangle$ are convergent and their limits are the same.

Therefore we can compose the definition

Definition 2. Consider the positive numbers $a$ and $b$. Then the common limit of the sequences $\left\langle a_{n}\right\rangle$ and $\left\langle b_{n}\right\rangle$ given by the above recursive way is called the arithmeticgeometric mean of $a$ and $b$, which is denoted by $A G(a, b)$.

It requires further analysis whether there exists such a formula, in possession of that one the arithmetic-geometric mean can be calculated directly for given $a$ and $b$. GAUSS proved in 1799 - at first for the pair $a=1, b=\sqrt{2}$, then generally - that the wanted formula exists but it is very unexpected:

$$
A G(a, b)=\left(\frac{2}{\pi} \int_{0}^{\frac{\pi}{2}} \frac{d \varphi}{\sqrt{a^{2} \cos ^{2} \varphi+b^{2} \sin \varphi}}\right)^{-1} .
$$

Let us take a historical digression by [4] in the interest of seeing from which follows this so-called elliptic integral. In the 17 th century two brothers, JOHANN and JAKOB BERNOULLI studied the following (,,isochrona paracentrica”) problem: which is the curve along this rolled down body that makes equal distances within equal times. Jakob Bernoulli got the equation

$$
\left(x^{2}+y^{2}\right)^{2}=2 a^{2}\left(x^{2}-y^{2}\right)
$$

and he called the curve lemniscus - see in Figure 5.

Jakob Bernoulli succeeded in finding an explicit formula for the calculation of arc length of the lemniscus, and it was the same which appeared by Gauss above.

More than three centuries later, Z. DARÓCZY and Zs. PÁlEs [6] constructed the theory which gave a significant help for examining this and similar iterations. They created the invariant equation

$$
F\left(K_{1}(a, b), K_{2}(a, b)\right)=F(a, b)
$$

where $F: I^{2} \rightarrow \mathbb{R}$ is the unknown function, and $K_{1}, K_{2}: I^{2} \rightarrow I$ denote known means of $a$ and $b$ of the interval $I$. They discovered that the only solution among the reflexive 


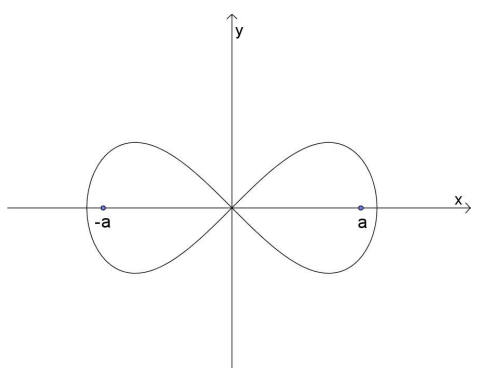

Figure 5

continuous functions is the mean, which is created in the above way with the help of $K_{1}$ and $K_{2}$. In this particular case it means that

$$
A G(A(a, b), G(a, b))=A G\left(\frac{a+b}{2}, \sqrt{a b}\right)=A G(a, b) \quad(a, b>0)
$$

which result played a very important role in giving the above explicit formula for the arithmetic-geometric mean.

We could mention here other Gauss iterations, in case of those the above result can easily be applied. For example, let us consider the following two sequences - see e.g. [5]:

$$
\begin{aligned}
a_{0}:=a>0 & b_{0}:=b>0 \\
a_{n+1} & :=\frac{a_{n}+b_{n}}{2} \quad b_{n+1}:=\frac{2 a_{n} b_{n}}{a_{n}+b_{n}} .
\end{aligned}
$$

This is similar to the above iteration, with the difference that the $(n+1)$-st term of the second sequence equals to the harmonic mean of the $n$-th terms of the sequences $\left\langle a_{n}\right\rangle$ and $\left\langle b_{n}\right\rangle$. With the help of the invariant equation, we get immediately the following

THEOREM 2. The sequences $\left\langle a_{n}\right\rangle$ and $\left\langle b_{n}\right\rangle$ are convergent and their limits are the same, namely $G(a, b)$.

Acknowledgement. The author is grateful to Professor Gyula Maksa for his significant help and to Anna Kozárné Fazekas and to Ildikó Bíró for reviewing and correcting the text of the paper. 


\section{References}

[1] J. Aczél, Lectures on Functional Equations and Their Applications, Vol. 19, Mathematics in Science and Engineering, Academic Press, New York-London, 1966.

[2] J. Aczél, On Applications and Theory of Functional Equations, Vol. 5, Elemente der Mathematik vom Höheren Standtpunkt aus, Birkhäuser Verlag, Basel/Stuttgart, 1969.

[3] A. Ambrus, Bevezetés a matematika-didaktikába, ELTE, Eötvös Kiadó, Budapest, 2004.

[4] Á. Besenyei, A számtani-mértani közép és egyéb érdekességek, KöMaL-Mathematical and Physical Journal for Secondary Schools (2009/2, 72-80 and 2009/3, 130-139), available at http: / /www. komal. hu.

[5] Z. Daróczy, Gaussian iteration of mean values and the existence of $\sqrt{2}$, Teaching Mathematics and Computer Sciences 1 (2003), 35-42.

[6] Z. Daróczy and Zs. Páles, Gauss-composition of means and the solution of the MatkowskiSuto problem, Publicationes Mathematicae Debrecen 61, no. 1-2 (2002), 157-218.

[7] S. Hajdu, Matematika 12., Múszaki Könyvkiadó, Budapest, 2005.

[8] J. Szikszai, A hatványközepek, Tankönyvkiadó, Budapest, 1987.

DOROTTYA BESSENYEI

TEACHER TRAINING SECONDARY GRAMMAR

SCHOOL OF DEBRECEN UNIVERSITY

H-4029, CSENGÖ STREET 4

DEBRECEN, HUNGARY

E-mail: nadasideklte-gyakorlo.sulinet.hu

(Received November, 2011) 